\title{
Fabrication of GaAs laser diodes on Si using low-temperature bonding of MBE-grown GaAs wafers with Si wafers
}

\author{
D. Cengher ${ }^{\mathrm{a}}$, Z. Hatzopoulos ${ }^{\mathrm{a}}, \mathrm{S}$. Gallis ${ }^{\mathrm{a}, 1}$, G. Deligeorgis ${ }^{\mathrm{a}}$, E. Aperathitis ${ }^{\mathrm{a}}$, \\ M. Androulidaki ${ }^{\mathrm{a}}$, M. Alexe ${ }^{\mathrm{b}}$, V. Dragoi ${ }^{\mathrm{b}, 2}$, E.D. Kyriakis-Bitzaros ${ }^{\mathrm{c}}$, \\ G. Halkias ${ }^{\mathrm{c}}$, A. Georgakilas ${ }^{\mathrm{a}, *}$ \\ a Physics Department, Microelectronics Research Group, FORTH, IESL and University Crete, P.O. Box 1527, \\ 711 10, Heraklion-Crete, Greece \\ ${ }^{\mathrm{b}}$ Max Planck Institute of Microstructure Physics, Weinberg 2, D-06120 Halle, Germany \\ ${ }^{\mathrm{c}}$ NCSR "Demokritos", Institute of Microelectronics, P.O. Box 60228, 15310 Agia Paraskevi, Greece
}

\begin{abstract}
We present a study of the properties of III-V structures integrated on Si by low-temperature GaAs-Si wafer bonding, using an intermediate spin-on-glass layer. Transmission electron microscopy revealed the good quality of the bonding and the absence of micro-cracks or dislocations in the semiconductor material. Photoluminescence measurements on $\mathrm{GaAs} / \mathrm{AlGaAs}$ multiple quantum well structures bonded on Si confirmed that the structural integrity of the quantum wells was preserved during the wafer bonding and thinning process. Photoreflectance measurements at temperatures in the range of $80-300 \mathrm{~K}$ showed that the bonded GaAs layers were practically stress free at room temperature, while a tensile stress appeared at lower temperature, due to the different thermal expansion coefficients of GaAs and Si. Laser devices with etched mirrors were fabricated on silicon and exhibited similar performances with reference devices fabricated on a GaAs substrate.
\end{abstract}

(C) 2002 Elsevier Science B.V. All rights reserved.

PACS: $81.05 . \mathrm{Ea} ; 81.15 . \mathrm{Jj}$

Keywords: A1. Characterization; A1. Interfaces; A1. Stresses; A1. Heterogeneous integration; B2. Semiconducting III-V materials; B3. Laser diodes

\footnotetext{
*Corresponding author. Tel.: +30-810394104; fax: +30810394106 .

E-mail address: alexandr@physics.uoc.gr (A. Georgakilas).

${ }^{1}$ Current address: CESTM, University at Albany, 251 Fuller Road, Albany, NY 12203, USA.

${ }^{2}$ Current address: EV Group, E. Thallner GmbH, Badhoering 26, 4780 Schaerding, Austria.
}

\section{Introduction}

The possibility to integrate GaAs devices with $\mathrm{Si}$ CMOS technology provides an interesting solution for optoelectronic and/or high-frequency-highpower applications. The heteroepitaxial growth of $\mathrm{GaAs}$ on $\mathrm{Si}$ [1] has been investigated to provide heterogeneous substrates including both Si and III$\mathrm{V}$ semiconductor layers. However, high dislocation 
density and stress, induced from lattice and thermal expansion mismatch, result in low performance and bad reliability of GaAs on Si laser diodes. We thus proposed [2] a new method to integrate, at the wafer scale, Si-based devices with III-V optoelectronic devices, which is based on low-temperature bonding of epitaxial GaAs wafers with CMOS wafers.

Unlike the reported [3] use of bonding agents requiring vacuum deposition techniques and hightemperature curing, we used a low-temperature spin-on-glass (SOG) layer, which can be uniformly applied over a wafer by spinning due to its low viscosity. In addition, the described process by London et al. [3] results to a thin foil of $\mathrm{Si}$ on a $\mathrm{GaAs}$ wafer and $\mathrm{Si}$ circuits must be processed afterwards. Our process, on the contrary, results in a thin III-V foil bonded on Si wafer using SOG as the bonding medium. The Si wafer can be a fully processed CMOS wafer, since the processing of III-V devices, which requires temperatures only up to $400 \mathrm{C}$ is made afterwards. Promising results for the low-temperature bonding of GaAs on Si using SOG have also been reported recently by Lin et al. [4] but no results were presented on the fabrication of bonded GaAs devices and, furthermore, this technique was not applied for wafer-scale integration.

In this paper, we present a study of the properties of III-V structures bonded on $\mathrm{Si}$ wafers with an intermediate SOG layer and report the fabrication of laser diodes with performance similar to devices fabricated on GaAs substrates.

\section{Material preparation}

Three-inch GaAs substrates were used to grow homoepitaxial layers of the required device structure as well as several test structures for the investigation of material characteristics. Structures to be bonded were grown in the inverse order of layers and a thin $(50-100 \mathrm{~nm})$ AlAs etch stop layer was included in order to facilitate substrate removal. A VG80H molecular beam epitaxy (MBE) system with an $\mathrm{As}_{4}$ source was used. A careful preparation of the MBE system resulted into complete elimination of "hillock type" oval defects [5].

The epitaxial GaAs wafer and a plain $\mathrm{Si}$ wafer were bonded face-to-face using a thin $(300 \mathrm{~nm})$ layer of SOG. Room-temperature bonding and low-temperature curing $\left(200^{\circ} \mathrm{C}\right)$ of SOG were used to avoid thermal degradation of the material as well as a thermally induced stress in the heterogeneous substrate because of the difference in thermal expansion coefficients.

Following bonding, the other side of GaAs substrate was initially mechanically thinned and finally dry etched in a reactive ion-etching (RIE) [2] reactor. The etching selectivity of GaAs over AlAs was 1000:1. This ensured no damage to the active layers until complete removal of the substrate material was achieved. A short wet etching step with $10 \% \mathrm{HF}$ was finally used to selectively remove the remaining AlAs etch stop layer. Thus the substrate is removed exposing the III-V active layers for processing of optoelectronic devices on the silicon wafer. Several samples were prepared using the above-mentioned technique. Finally, laser diodes with etched mirrors [6] were processed using similar structures on GaAs and $\mathrm{Si}$ substrates, to evaluate the quality of the GaAs/ $\mathrm{SOG} / \mathrm{Si}$ bonded material.

\section{Results and discussion}

The average interface energy of the bonded GaAs/silicon surfaces was measured using the crack opening method by inserting a blade between the bonded wafers. The interface energy of the as-bonded structure was $0.46 \mathrm{~J} / \mathrm{m}^{2}$ and reached the value of $1.4 \mathrm{~J} / \mathrm{m}^{2}$ after annealing at $200^{\circ} \mathrm{C}$.

The amount of bowing of the bonded GaAs/ silicon wafers was measured during this annealing step. It was approximately $500 \mu \mathrm{m}$ at $200^{\circ} \mathrm{C}$, but reached zero value after cooling down to room temperature. The wafer bowing was free of hysteresis effects, perfectly symmetric and equal for the two wafers. This bow is associated with stress in both wafers and the results indicate that the GaAs/silicon lattice correlation is basically established at room temperature and remains 
unaffected by the annealing. The two bonded materials are stress free at room temperature.

Transmission electron microscopy (TEM) observations of cross-sectional specimens revealed a continuous layer of SOG material in contact with $\mathrm{Si}$ as well as GaAs material. Fig. 1 presents a TEM micrograph of the GaAs/SOG/silicon sample and we can see that the interfaces are very good and the GaAs layer is free of crystalline defects due to the bonding process. For comparison, the GaAs grown epitaxially on $\mathrm{Si}$ was reported to have a high dislocation density [1] of the order of $10^{8}$ dislocations $/ \mathrm{cm}^{2}$.

Photoluminescence (PL) measurements on GaAs/AlGaAs multiple quantum well (MQW) structures bonded on $\mathrm{Si}$ were used to evaluate the compatibility of the integration (bonding, thinning) process with the structural integrity of MQW heterostructures. Fig. 2 presents the $20 \mathrm{~K}$ PL spectrum of a structure consisting of five quantum wells with thicknesses of $3.8,5.0,7.0$, 10.0 and $19.0 \mathrm{~nm}$, separated by $50 \mathrm{~nm} \mathrm{Al}_{0.26-}$ $\mathrm{Ga}_{0.74} \mathrm{As}$ barriers. Clear emission from all quantum wells was recorded similarly to a reference structure on a GaAs substrate. However, some PL peaks recorded at temperatures between 200 and $80 \mathrm{~K}$ showed emission lines splitting. This is attributed to valence band splitting and indicates the presence of a biaxial strain in the material at these temperatures.

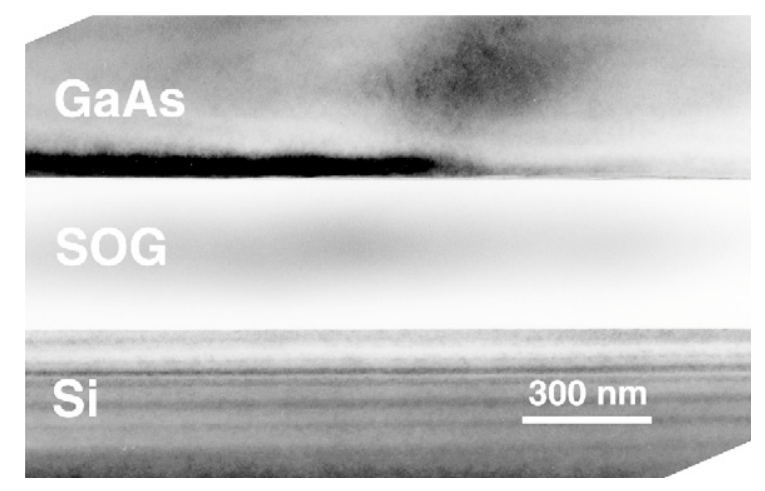

Fig. 1. TEM cross-sectional micrograph, viewed along $\langle 110\rangle$ $\mathrm{Si}$ zone axis, showing the interfacial region of a $\mathrm{GaAs} / \mathrm{SOG} / \mathrm{Si}$ sample.

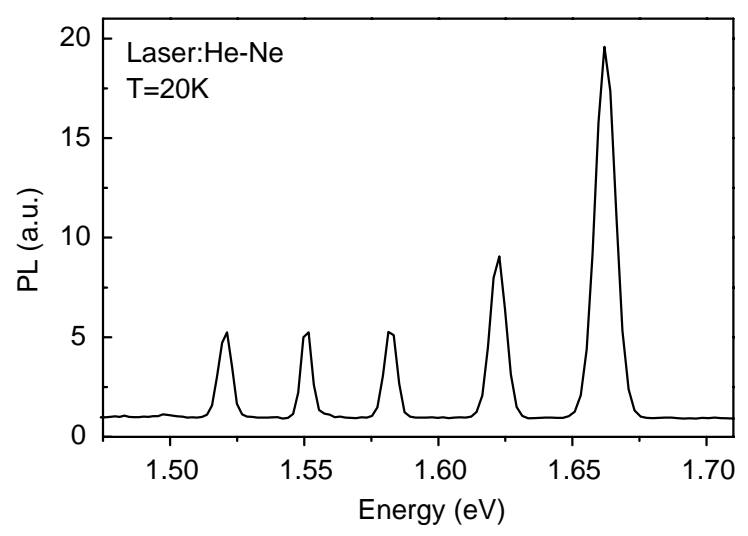

Fig. 2. PL spectrum at $20 \mathrm{~K}$ for a bonded MQW structure consisting of five quantum wells with thicknesses of 3.8, 5.0, 7.0, 10.0 and $19.0 \mathrm{~nm}$ separated by $50 \mathrm{~nm} \mathrm{Al}_{0.26} \mathrm{Ga}_{0.74} \mathrm{As}$ barriers.

A comparative study of biaxial stress as a function of temperature, in the range of 80 $300 \mathrm{~K}$, for a $2 \mu \mathrm{m}$-thick GaAs film bonded on an $\mathrm{Si}$ wafer (GaAs/SOG/Si sample \#133) and a similar film grown by $\mathrm{MBE}$ on an $\mathrm{Si}\left(\begin{array}{lll}0 & 0 & 1\end{array}\right)$ substrate (sample \#66) was carried out using photoreflectance (PR) measurements.

A conventional experimental set-up was used for the PR measurements [7]. The line shapes of the PR spectra (Fig. 3) were modeled using Eq. (1), according to Aspnes theory [8]:

$\frac{\Delta R}{R}=\sum_{j=1}^{f} \frac{A_{j} \cos \left[\Phi_{j}+2.5 \operatorname{ArcCos}\left(\kappa_{j}\right)\right]}{\left(\left(E-E_{\mathrm{g} j}\right)^{2}+\Gamma_{j}^{2}\right)^{1.25}}$,

where $f$ is the number of the transitions to be fitted, $A$ is the amplitude, $\Phi$ is a phase angle (interference effects), $E$ is the photon energy, $E_{\mathrm{g}}$ is the energy bandgap, $\Gamma$ is the broadening parameter due to carrier scattering processes and $\kappa$ is the phase angle of the complex number $\left[\left(E-E_{\mathrm{g}}\right)-\mathrm{i} \Gamma\right]$. Three-dimensional one-electron optical transitions were assumed. For a given transition, the fitting parameters were $\dot{A}, \Phi, E_{\mathrm{g}}$ and $\Gamma$ and Fig. $3 b$ is a typical result of the fitting of the PR spectra using Eq. (1).

Fig. 3a shows the experimental PR spectra of the $\mathrm{GaAs} / \mathrm{SOG} / \mathrm{Si}$ (\#133) and $\mathrm{GaAs} / \mathrm{Si}$ (\#66) samples at $250 \mathrm{~K}$. Arrows have been inserted in Fig. 3 to indicate the bandgap values, as follows: $E_{1(133)}=$ energy bandgap of sample \#133, 

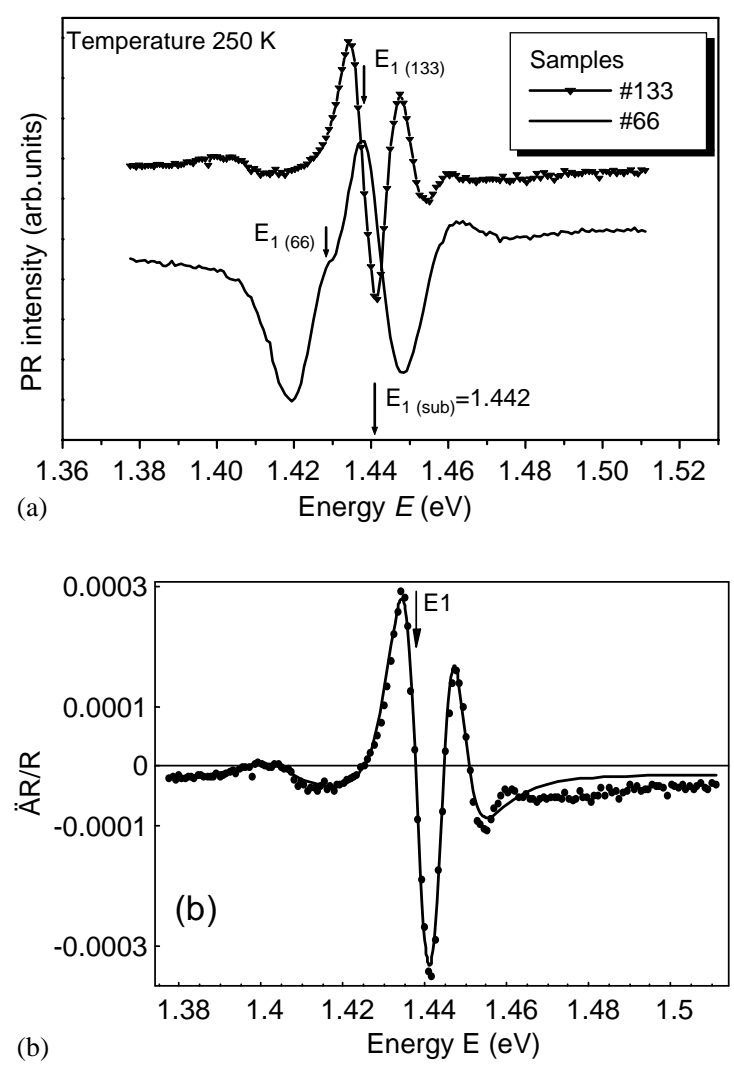

Fig 3. (a) Experimental PR spectra at $250 \mathrm{~K}$ for $2 \mu \mathrm{m}$ GaAs films of heteroepitaxial $\mathrm{GaAs} / \mathrm{Si}$ (\#66) and bonded $\mathrm{GaAs} / \mathrm{Si}$ (\#133), revealing a lower tensile strain in the bonded film. (b) Theoretical fit of the experimental $250 \mathrm{~K}$ PR spectrum of sample \#133. ( $E_{1(133)}$ arrow indicates the bandgap).

$E_{1(66)}=$ energy bandgap of sample \#66, $E_{1(\text { sub })}=$ energy bandgap of GaAs (reference bulk substrate). From the fit of Eq. (1) to the PR spectra, the energy positions of the first transition $\left(E_{1}\right)$ and second transition $\left(E_{2}\right)$ were determined for the bonded and epitaxial GaAs-on-Si samples. Since the energy position of the transition $\left(E_{1}\right)$ corresponds to the energy bandgap of each sample, the energy difference between this transition and the value of the bandgap of the GaAs substratewhich was also obtained at each temperature by fitting the PR line shapes-corresponds to the energy bandgap change due to the biaxial strain. From the calculated bandgap shift, the internal strain $(\varepsilon)$ in samples \#133 and \#66 could be determined from elastic theory [9]. Fig. 4a presents
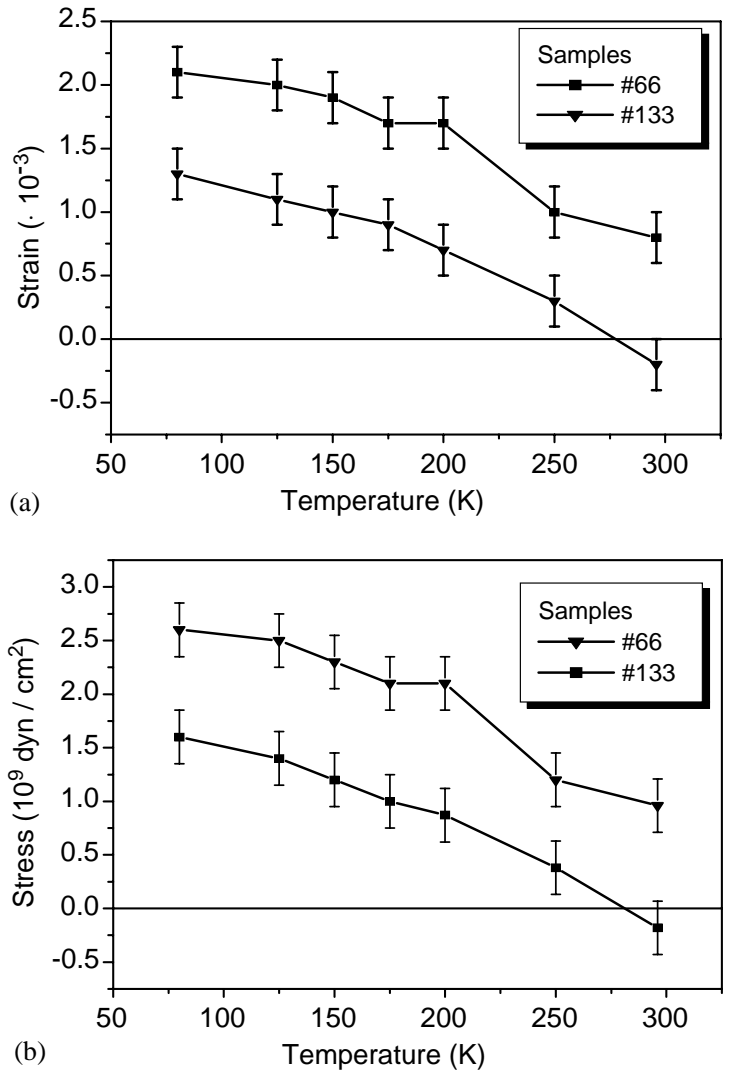

Fig 4. (a) Biaxial strain and (b) stress in the $2 \mu \mathrm{m}$ GaAs films of bonded $\mathrm{GaAs} / \mathrm{Si}$ (\#133) and heteroepitaxial GaAs/Si (\#66), in the temperature range of $80-296 \mathrm{~K}$. Error bars for strain are $\pm 0.2 \times 10^{-3}$ and for stress are $\pm 2.5 \times 10^{8} \mathrm{dyn} / \mathrm{cm}^{2}$.

the dependence of internal strain $\varepsilon$ on the temperature.

From the determined strain $\varepsilon$, the stress $\sigma$ can also be calculated [9]. The results for the stress $(\sigma)$ have been plotted versus temperature in Fig. $4 \mathrm{~b}$ for both the $2 \mu \mathrm{m} \mathrm{GaAs} / \mathrm{Si}$ (\#66) and $2 \mu \mathrm{m} \mathrm{GaAs} / \mathrm{SOG} /$ $\mathrm{Si}$ (\#133) samples. The stress of the GaAs/SOG/Si sample was found to be slightly compressive at room temperature but it must be pointed out that the estimated small value of compressive stress is within the accuracy of the calculation method. The comparison with the epitaxial GaAs film indicates clearly that the bonded film is essentially strain-free at room temperature and this is very promising for the reliability of laser diodes that can be processed from bonded GaAs material. 
Finally, as shown in Fig. 4b a similar increase of the biaxial stress occurred as a function of decreasing temperature in both the bonded $\mathrm{GaAs} / \mathrm{SOG} / \mathrm{Si}$ and the epitaxial GaAs-on-Si samples. This indicates that in both cases the tensile stress is a thermal stress created by the different thermal expansion coefficients of GaAs and Si.

\section{Laser devices bonded on silicon}

Laser devices with etched mirrors were fabricated [6] from a separate confinement 4QW AlGaAs/GaAs heterostructure bonded on $\mathrm{Si}$ and the results were compared with those for a similar structure grown on semi-insulating (SI) GaAs substrate.

Fig. 5 presents characterization results for a laser device fabricated on the bonded structure and for a similar laser device fabricated on the reference GaAs wafer. The measurements were performed at room temperature, $1.0 \mathrm{kHz}$ frequency and at a low-duty cycle of $0.5 \times 10^{-3}$, to reduce the effect of the device heating. Similar device performances were observed. The results prove that laser diode devices can be processed on the bonded material. This means that the bonded material can withstand the device fabrication

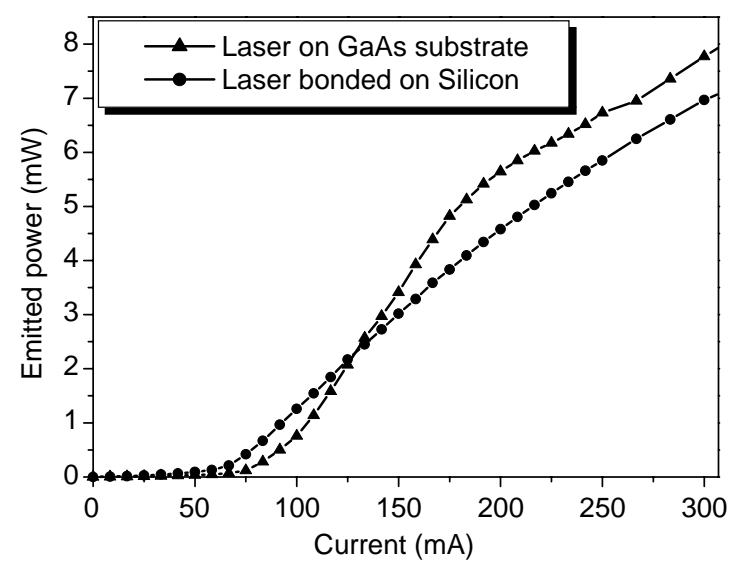

Fig 5. Comparison of a laser with RIE etched mirrors fabricated on bonded $\mathrm{GaAs} / \mathrm{SOG} / \mathrm{Si}$ and a reference laser fabricated on SI GaAs substrate. process and that the bonded GaAs material quality is not degraded by the formation of crystalline defects. Some apparent differences in the characteristics of the lasers on the $\mathrm{Si}$ and $\mathrm{GaAs}$ substrates were related only to variations in the quality of the RIE-formed mirrors.

Measurements at high-duty cycle, of $22.2 \times 10^{-3}$, where device heating becomes important, showed that both devices reach the thermal rollover point. This rollover was not observed at low-duty cycle measurements even for currents up to $600 \mathrm{~mA}$, i.e., $9.5 I_{\mathrm{th}}$, for the device fabricated on silicon. The rollover point was at $425 \mathrm{~mA}$ for the laser device on silicon, while it was approximately $12.2 \%$ lower for the reference laser device on the GaAs substrate. This difference corresponds to a difference in the electrical peak power generated in the junction region of approximately $4 \mathrm{~kW} / \mathrm{cm}^{2}$ and indicates that the thermal resistance of the device bonded on silicon with the thin SOG layer was lower than that of the device epitaxially grown on the GaAs substrate due to the higher thermal conductivity of Si.

\section{Conclusions}

An alternative to the epitaxial growth approach for $\mathrm{GaAs} / \mathrm{Si}$ wafer-scale integration has been developed and evaluated. It is based on lowtemperature bonding of GaAs and $\mathrm{Si}$ wafers using an intermediate SOG layer. High-quality heterogeneous substrates have been produced, as it is evident from the optical and structural characterization. Strain measurements showed that the bonded III-V layers were stress free at room temperature. The bonded structures could withstand all the processing steps required in the fabrication of optoelectronic devices and the laser diodes on $\mathrm{Si}$ exhibited improved thermal behavior.

\section{Acknowledgements}

EU supported this work through ESPRIT project no. 28998 “BONTEC”. Author D. Cengher would like also to acknowledge support from 
Marie-Curie Training Site Fellowships Program, contract no. HPMT-GH-00-00177.

\section{References}

[1] C.B. Lioutas, A. Delimitis, A. Georgakilas, Thin Solid Films 336 (1998) 96.

[2] A. Georgakilas, M. Alexe, G. Deligeorgis, D. Cengher, E. Aperathitis, M. Androulidaki, S. Gallis, Z. Hatzopoulos, G. Halkias, IEEE CAS 2001 Conference Proceedings 01TH8547, 2001, p. 239.
[3] J.M. London, A.H. Loomis, J.F. Ahadian, C.G. Fonstad, IEEE Photon. Technol. Lett. 11 (1999) 958.

[4] H.C. Lin, K.L. Chang, G.W. Pickrell, K.C. H4sieh, K.Y. Cheng, J. Vac. Sci. Technol. B 20 (2002) 752.

[5] Z. Hatzopoulos, D. Cengher, G. Deligeorgis, M. Androulidaki, E. Aperathitis, G. Halkias, A. Georgakilas, J. Crystal Growth 227-8 (2001) 193.

[6] E. Aperathitis, D. Cengher, M. Kayambaki, M. Androulidaki, G. Deligeorgis, K. Tsagaraki, Z. Hatzopoulos, A. Georgakilas, Mater. Sci. Eng. B80 (2001) 77.

[7] R.K. Willardson, A.C. Beer, Modulation Tech. 9 (1972).

[8] D.E. Aspnes, Surf. Sci. 37 (1973) 418.

[9] A. Gavini, M. Cardona, Phys. Rev. B 1 (1970) 672. 